\title{
Gauss-Legendre Iterative Methods and Their Applications on Nonlinear Systems and BVP-ODEs
}

\author{
Zhongli Liu ${ }^{*}$, Guoqing Sun ${ }^{2}$ \\ ${ }^{1}$ College of Biochemical Engineering, Beijing Union University, Beijing, China \\ ${ }^{2}$ College of Renai, Tianjin University, Tianjin, China \\ Email: shtzhongli@buu.edu.cn, sunguoqing66@126.com
}

How to cite this paper: Liu, Z.L. and Sun, G.Q. (2016) Gauss-Legendre Iterative Methods and Their Applications on Nonlinear Systems and BVP-ODEs. Journal of Applied Mathematics and Physics, 4, 2038-2046. http://dx.doi.org/10.4236/jamp.2016.411203

Received: October 24, 2016

Accepted: November 15, 2016

Published: November 18, 2016

Copyright (c) 2016 by authors and Scientific Research Publishing Inc. This work is licensed under the Creative Commons Attribution International

License (CC BY 4.0).

http://creativecommons.org/licenses/by/4.0/

\begin{abstract}
In this paper, a group of Gauss-Legendre iterative methods with cubic convergence for solving nonlinear systems are proposed. We construct the iterative schemes based on Gauss-Legendre quadrature formula. The cubic convergence and error equation are proved theoretically, and demonstrated numerically. Several numerical examples for solving the system of nonlinear equations and boundary-value problems of nonlinear ordinary differential equations (ODEs) are provided to illustrate the efficiency and performance of the suggested iterative methods.
\end{abstract}

\section{Keywords}

Iterative Method, Gauss-Legendre Quadrature Formula, Nonlinear Systems, Third-Order Convergence, Nonlinear ODEs

\section{c) (i) Open Access}

\section{Introduction}

We consider the general form of a system of nonlinear equations as follows:

$$
F(x)=\left(f_{1}(x), f_{2}(x), \cdots, f_{n}(x)\right)^{\mathrm{T}}=0,
$$

where $F: D \subseteq R^{n} \rightarrow R^{n}$ is a given nonlinear vector function, and each function $f_{i}(x)$ can be thought of as mapping of a vector $x=\left(x_{1}, x_{2}, \cdots, x_{n}\right)^{\mathrm{T}}$, which is the $n$ dimensional space $R^{n}$ into the real number $R$.

Numerical solutions for systems of nonlinear equations have always appealed greatly to people in scientific computation and engineering fields. Some boundary-value problems of nonlinear ordinary differential equations (ODEs) can be transformed to nonlinear systems like (1) by the finite difference method. Constructing an efficiently iterative method to approximate the root $\xi \in D$ (such that $F(\xi)=0$ ) of Equation (1) is a 
typical and important issue in nonlinear numerical computation. The Newton's method (see [1] [2]) is one of the widely used methods for solving nonlinear equations by iteration as follows:

$$
x_{k+1}=x_{k}-F^{\prime}\left(x_{k}\right)^{-1} F\left(x_{k}\right), \quad k=0,1,2, \cdots,
$$

where $F^{\prime}(x)^{-1}$ is the inverse of first Fréchet derivative $F^{\prime}(x)$ which is a Jacobian matrix of the nonlinear vector function $F(x)$. It converges quadratically when an initial guess value $x_{0}$ is close to the root $\xi$ of Equation (1). In recent years, several iterative methods have been used to solve nonlinear equations and systems of nonlinear equations. In order to improve the order of convergence, a few two-step variants of Newton's methods with cubic convergence have been proposed in some literature [3]-[11] and references therein for solving systems of nonlinear equations. S. We era-

koon and T. Fernando [5] using the Newton theorem $f(x)=f(x)_{n}+\int_{x_{n}}^{x} f^{\prime}(t) \mathrm{d} t$, proposed the Newton-type method with third-order convergence for nonlinear equation and systems. M. Darvishi and A. Barati [6] received a third-order convergence iterative method based on Adomian decomposition method to the systems of nonlinear equations. M. Frontini and E. Sormani [7] presented third-order midpoint-methods using numerical quadrature formula. A. Cordero and J. R. Torregrsa [8] developed third-order convergence Newton-Simpson's method and Open Newton's method using the simple Simpson's rule and an open quadrature formula of high order respectively. These are all classic two-step Newton-type methods to approximate the root of a system of nonlinear equations.

In Section 2 of this paper, we propose a group of two-step iterative methods with third-order convergence by Gauss-Legendre quadrature formula [12]:

$$
\int_{a}^{b} f(x) \mathrm{d} x=\frac{b-a}{2} \sum_{i=1}^{n} w_{i} f\left(\frac{a+b}{2}+\frac{b-a}{2} t_{i}\right),
$$

where the necessary symmetrical conditions is

$$
\sum w_{i}=2, \sum t_{i}=0, \sum w_{i} t_{i}=0 .
$$

Several numerical examples are provided in Section 3 using Gauss-Legendre iterative method for solving systems of nonlinear equations and boundary-value problems of nonlinear ODEs, and we finally make conclusions in Section 4.

\section{The Iterative Methods and Cubic Convergence}

Assume $F: D \subset R^{n} \rightarrow R^{n}$ be a Fréchet differentiable function in a convex $D \subset R^{n}$. We have the mean-value theorem of multivariable vectors function $F(x)$ in [1]:

$$
F(x)-F\left(x_{k}\right)=\int_{0}^{1} F^{\prime}\left(x_{k}+t\left(x-x_{k}\right)\right)\left(x-x_{k}\right) \mathrm{d} t,
$$

Using the left rectangular integral rule:

$$
\int_{0}^{1} F^{\prime}\left(x_{k}+t\left(x-x_{k}\right)\right)\left(x-x_{k}\right) \mathrm{d} t \approx F^{\prime}\left(x_{k}\right)\left(x-x_{k}\right),
$$

And by $F(x)=0$, we can get Newton's Method (2). 
Now, we apply the Gauss-Legendre quadrature formula (3) to approximate the integral on the right side of Equation (5), that is,

$$
\int_{0}^{1} F^{\prime}\left(x_{k}+t\left(x-x_{k}\right)\right)\left(x-x_{k}\right) \mathrm{d} t \approx \frac{1}{2} \sum_{i=1}^{n} w_{i} F^{\prime}\left(\frac{x_{k}+x}{2}+\frac{x-x_{k}}{2} t_{i}\right)\left(x-x_{k}\right),
$$

and using $F(x)=0$ we have

$$
x-x_{k} \approx-2\left[\sum_{i=1}^{n} w_{i} F^{\prime}\left(\frac{x_{k}+x}{2}+\frac{x-x_{k}}{2} t_{i}\right)\right]^{-1} F\left(x_{n}\right) .
$$

Herein, taking $x$ as the next iterative step of $x_{k}$, we get the following implicit structure:

$$
x_{k+1}=x_{k}-2\left[\sum_{i=1}^{n} w_{i} F^{\prime}\left(\frac{x_{k}+x_{k+1}}{2}+\frac{x_{k+1}-x_{k}}{2} t_{i}\right)\right]^{-1} F\left(x_{n}\right) .
$$

We use the $k$-th iteration $y_{k}$ of the Newton's method to replace $x_{k+1}$ on the right side of the expression (9), and we construct the following group of two-step iterative schemes:

$$
\left\{\begin{array}{l}
y_{k}=x_{k}-F^{\prime}\left(x_{k}\right)^{-1} F\left(x_{k}\right) \\
x_{k+1}=x_{k}-2\left[\sum_{i=1}^{n} w_{i} F^{\prime}\left(\frac{x_{k}+y_{k}}{2}+\frac{y_{k}-x_{k}}{2} t_{i}\right)\right]^{-1} F\left(x_{k}\right)
\end{array} .\right.
$$

We state and prove the convergence theorem for the schemes (10) as follows:

Theorem Let $F: D \subset R^{n} \rightarrow R^{n}$ be a $k$-time Fréchet differentiable function in a convex set $D$ with a root $\xi \subset D$ and $x_{0}$ be close to $\xi$, then the group of iterative methods (10) is cubically convergent, and the error equation is

$$
e_{k+1}=\left(C_{2}^{2}-C_{3}+\frac{3}{8}\left(2+\sum_{i=1}^{n} w_{i} t_{i}^{2}\right) C_{3}\right) e_{k}^{3}+O\left(e_{k}^{4}\right) .
$$

Proof. As $F(\xi)=0$, and noting that $x_{k}-\xi=e_{k}, C_{k}=F^{\prime}(\xi)^{-1} \frac{F^{(k)}(\xi)}{k !}$.

By Taylor's expansion, we have

$$
\begin{aligned}
F\left(x_{k}\right) & =F^{\prime}(\xi)\left(x_{k}-\xi\right)+\frac{1}{2 !} F^{\prime \prime}(\xi)\left(x_{k}-\xi\right)^{2}+\frac{1}{3 !} F^{\prime \prime \prime}(\xi)\left(x_{k}-\xi\right)^{3}+O\left(x_{k}-\xi\right)^{4} \\
& =F^{\prime}(\xi)\left[e_{k}+C_{2} e_{k}^{2}+C_{3} e_{k}\right]+O\left(e_{k}^{4}\right),
\end{aligned}
$$

and

$$
F^{\prime}\left(x_{k}\right)=F^{\prime}(\xi)\left[I+2 C_{2} e_{k}+3 C_{3} e_{k}^{2}+O\left(e_{k}^{3}\right)\right] .
$$

Then, suppose that

$$
F^{\prime}\left(x_{k}\right)^{-1}=\left[D\left(e_{k}\right)^{-1}\right] F^{\prime}(\xi)^{-1}
$$

where

$$
D\left(e_{k}\right)=I+2 C_{2} e_{k}+3 C_{3} e_{k}^{2}+O\left(e_{k}^{3}\right)
$$

The inverse of $D\left(e_{k}\right)$ is given by 


$$
D\left(e_{k}\right)^{-1}=I+K_{1} e_{k}+K_{2} e_{k}^{2},
$$

where $K_{1}$ and $K_{2}$ will be satisfied the definition of the following inversion.

$$
D\left(e_{k}\right) D\left(e_{k}\right)^{-1}=D\left(e_{k}\right)^{-1} D\left(e_{k}\right)=I .
$$

That is

$$
\left(I+2 C_{2} e_{n}+3 C_{3} e_{n}^{2}\right)\left(I+K_{1} e_{n}+K_{2} e_{n}^{2}\right)=I .
$$

From (17), we have

$$
K_{1}=-2 C_{2}, K_{2}=4 C_{2}^{2}-3 C_{3} .
$$

Therefore,

$$
F^{\prime}\left(x_{k}\right)^{-1}=\left[I-2 C_{2} e_{k}+\left(4 C_{2}^{2}-3 C_{3}\right) e_{k}^{2}+\cdots\right] F^{\prime}(\xi)^{-1} .
$$

And

$$
\begin{aligned}
F^{\prime}\left(x_{k}\right)^{-1} F\left(x_{k}\right) & =\left[I-2 C_{2} e_{k}+\left(4 C_{2}^{2}-3 C_{3}\right) e_{k}^{2}+\cdots\right] \times\left(e_{k}+C_{2} e_{k}^{2}+C_{3} e_{k}^{3}+\cdots\right) \\
& =e_{k}-C_{2} e_{k}^{2}+\left(2 C_{2}^{2}-2 C_{3}\right) e_{k}^{3}+O\left(e_{k}^{4}\right) .
\end{aligned}
$$

By the first step of (10), we have

$$
y_{k}=\xi+C_{2} e_{k}^{2}+2\left(C_{3}-C_{2}^{2}\right) e_{k}^{3}+O\left(e_{k}^{4}\right)=\xi+\Delta,
$$

Here, suppose $\Delta=C_{2} e_{k}^{2}+2\left(C_{3}-C_{2}^{2}\right) e_{k}^{3}+O\left(e_{k}^{4}\right)$.

So,

$$
\begin{gathered}
\frac{x_{k}+y_{k}}{2}=\xi+\frac{1}{2} x_{k}+\frac{1}{2} \Delta=\xi+\frac{1}{2} e_{k}+\frac{1}{2} \Delta, \\
\frac{y_{k}-x_{k}}{2}=\frac{\xi}{2}+\frac{1}{2} \Delta-\frac{1}{2}\left(\xi+e_{k}\right)=-\frac{1}{2} e_{k}+\frac{1}{2} \Delta .
\end{gathered}
$$

Furthermore,

$$
\frac{x_{k}+y_{k}}{2}+\frac{y_{k}-x_{k}}{2} t_{i}=\xi+\frac{1}{2}\left(1-t_{i}\right) e_{k}+\frac{1}{2}\left(1+t_{i}\right) \Delta=\xi+d_{k},
$$

where,

$$
d_{k}=\frac{1}{2}\left(1-t_{i}\right) e_{k}+\frac{1}{2}\left(1+t_{i}\right) \Delta .
$$

Then,

$$
\begin{aligned}
& F^{\prime}\left(\frac{x_{k}+y_{k}}{2}+\frac{y_{k}-x_{k}}{2} t_{i}\right) \\
= & F^{\prime}(\xi)\left(I+2 C_{2} d_{k}+3 C_{3} d_{k}^{2}+\cdots\right) \\
= & F^{\prime}(\xi)\left[I+C_{2}\left(1-t_{i}\right) e_{k}+C_{2}\left(1+t_{i}\right) \Delta+\frac{3}{4} C_{3}\left(1-t_{i}\right)^{2} e_{k}^{2}+O\left(e_{k}^{3}\right)\right] \\
= & F^{\prime}(\xi)\left[I+C_{2}\left(1-t_{i}\right) e_{k}+C_{2}^{2}\left(1+t_{i}\right) e_{k}^{2}+\frac{3}{4} C_{3}\left(1-t_{i}\right)^{2} e_{k}^{2}+O\left(e_{k}^{3}\right)\right] .
\end{aligned}
$$

Let 


$$
\begin{aligned}
B & =\sum_{i=1}^{n} w_{i} F^{\prime}\left(\frac{x_{k}+y_{k}}{2}+\frac{y_{k}-x_{k}}{2} t_{i}\right) \\
& =\sum_{i=1}^{n} w_{i} F^{\prime}(\xi)\left[I+C_{2}\left(1-t_{i}\right) e_{k}+C_{2}^{2}\left(1+t_{i}\right) e_{k}^{2}+\frac{3}{4} C_{3}\left(1-t_{i}\right)^{2} e_{k}^{2}+O\left(e_{k}^{3}\right)\right] \\
& =F^{\prime}(\xi)\left[\sum_{i=1}^{n} w_{i} I+C_{2} e_{k} \sum_{i=1}^{n} w_{i}\left(1-t_{i}\right)+C_{2}^{2} e_{k}^{2} \sum_{i=1}^{n} w_{i}\left(1+t_{i}\right)+\frac{3}{4} C_{3} e_{k}^{2} \sum_{i=1}^{n} w_{i}\left(1-2 t_{i}+t_{i}^{2}\right)+O\left(e_{k}^{3}\right)\right] .
\end{aligned}
$$

By the conditions (4), we have

$$
B=F^{\prime}(\xi)\left[2 I+2 C_{2} e_{k}+2 C_{2}^{2} e_{k}^{2}+\frac{3}{4} C_{3} e_{k}^{2}\left(2+\sum_{i=1}^{n} w_{i} t_{i}^{2}\right)+O\left(e_{k}^{3}\right)\right] .
$$

So the iterative schemes (10) can be written as $x_{k+1}=x_{k}-2 B^{-1} F\left(x_{k}\right)$. Subtracting $\xi$ from both sides of this equation, we can obtain

$$
\frac{1}{2} B e_{k+1}=\frac{1}{2} B e_{k}-F\left(x_{k}\right) \text {, }
$$

that is

$$
\begin{aligned}
\left(I+C_{2} e_{k}+O\left(e_{k}^{2}\right)\right) e_{k+1} & =e_{k}+C_{2} e_{k}^{2}+C_{2}^{2} e_{k}^{3}+\frac{3}{8} C_{3} e_{k}^{3}\left(2+\sum_{i=1}^{n} w_{i} t_{i}^{2}\right)+O\left(e_{k}^{4}\right)-F\left(x_{k}\right) \\
& =\left(C_{2}^{2}-C_{3}\right) e_{k}^{3}+\frac{3}{8} C_{3}\left(2+\sum_{i=1}^{n} w_{i} t_{i}^{2}\right) e_{k}^{3}+O\left(e_{k}^{4}\right)
\end{aligned}
$$

Therefore, the error equation is

$$
e_{k+1}=\left(C_{2}^{2}-C_{3}+\frac{3}{8}\left(2+\sum_{i=1}^{n} w_{i} t_{i}^{2}\right) C_{3}\right) e_{k}^{3}+O\left(e_{k}^{4}\right) .
$$

This shows that the group of iterative methods (10) is third-order convergent.

As for the iterative methods (10), when $n=1, w_{1}=2, t_{1}=0$, then it can be transformed to the following form:

$$
\left\{\begin{array}{l}
y_{k}=x_{k}-F^{\prime}\left(x_{k}\right)^{-1} F\left(x_{k}\right) \\
x_{k+1}=x_{k}-F^{\prime}\left(\frac{x_{k}+y_{k}}{2}\right)^{-1} F\left(x_{k}\right)
\end{array} .\right.
$$

When $n=2, w_{1}=w_{2}=1, t_{1}=\mu, t_{2}=-\mu, \mu \in R$ is a parameter, then the methods (10) can be written as follows:

$$
\left\{\begin{array}{l}
y_{k}=x_{k}-F^{\prime}\left(x_{k}\right)^{-1} F\left(x_{k}\right) \\
x_{k+1}=x_{k}-2\left[\sum_{i=1}^{n} F^{\prime}\left(\frac{x_{k}+y_{k}}{2}-\frac{y_{k}-x_{k}}{2} \mu\right)+F^{\prime}\left(\frac{x_{k}+y_{k}}{2}+\frac{y_{k}-x_{k}}{2} \mu\right)\right]^{-1} F\left(x_{k}\right)
\end{array} .\right.
$$

Especially, we take the parameter $\mu=0.577350$ in the iterative method (33) to make applications in the following section.

\section{Numerical Examples}

The iterative method (33) with $\mu=0.577350$ is demonstrated for solving systems of nonlinear equations and some two-point boundary-value problems of nonlinear ODEs. 
Example 1. Consider a system of nonlinear equations with variables $n=5$ :

$$
\sum_{j=1, j \neq i}^{n} x_{j}-(n-1) x_{i}^{2}=0, \quad 1 \leq i \leq n,
$$

where $x_{0}=(2,2,2,2,2)^{\prime}$ is the guess value. We can get the exact solution $\xi=(1,1,1,1,1)^{\prime}$ by the iterative method (33) for Equation (34). The numerical results are shown in Table 1 , in which $k$ refers to the number of iterations.

Example 2. Consider solving the following two-point boundary-value problem of nonlinear ODE:

$$
\left\{\begin{array}{l}
x^{\prime \prime}(t)+\sqrt{x(t)}=0, \quad t \in(0,1) \\
x(0)=0, \quad x(1)=1
\end{array}\right.
$$

We discretize the nonlinear ODE (35) with the finite difference method. Taking nodes $t_{i}=i h$, where $h=1 / N$ and $N=10$ herein, we can obtain the following system of nine-variables nonlinear equations:

$$
\left\{\begin{array}{l}
2 x_{1}-h^{2} \sqrt{x_{1}}-x_{2}=0 \\
-x_{i-1}+2 x_{i}-h^{2} \sqrt{x_{i}}-x_{i+1}=0, i=2,3, \cdots, 8, \\
-x_{8}+2 x_{9}-1-h^{2} \sqrt{x_{9}}=0
\end{array}\right.
$$

where $x_{0}=(1,1,1,1,1,1,1,1,1)^{\prime}$. Using the iterative method (33), we get the numerical solutions $\xi$ of Equation (36), that is,

$$
\begin{aligned}
\xi= & (0.1283710267934016 \cdots, 0.2531591633178528 \cdots, 0.37291580738666021 \cdots, \\
& 0.4865657681707311 \cdots, 0.5932403023143547 \cdots, 0.6922126270196785 \cdots, \\
& 0.7828650200725204 \cdots, 0.8646694471440053 \cdots, 0.9371751138295893 \cdots)^{\prime} .
\end{aligned}
$$

The numerical results for the system of nonlinear Equations (36) derived from ODE (35) are shown in Table 2.

According to results of the above two numerical experiments, the iterative method (33) can achieve third-order convergence for systems of nonlinear equations, and their numerical solutions show also the method is feasible.

Table 1. The numerical solutions and errors of the system of Equation (34) using the method (33).

\begin{tabular}{ccccccc}
\hline$k$ & 1 & 2 & 3 & 4 & 5 & 6 \\
\hline$\left\|x_{k}-\xi\right\|_{2}$ & $3.1943 \mathrm{e}-1$ & $4.3758 \mathrm{e}-3$ & $1.6660 \mathrm{e}-8$ & $9.2481 \mathrm{e}-25$ & $1.5819 \mathrm{e}-73$ & $7.9180 \mathrm{e}-220$ \\
$\left\|F\left(x_{k}\right)\right\|_{2}$ & $1.4602 \mathrm{e}-0$ & $1.7537 \mathrm{e}-2$ & $6.6640 \mathrm{e}-8$ & $3.6992 \mathrm{e}-24$ & $6.3278 \mathrm{e}-73$ & $3.1672 \mathrm{e}-219$ \\
\hline
\end{tabular}

Table 2. The numerical solutions and errors of the system of nonlinear Equation (36).

\begin{tabular}{ccccc}
\hline$k$ & 1 & 2 & 3 & 4 \\
\hline$\left\|x_{k}-\xi\right\|_{2}$ & $4.9234 \mathrm{e}-4$ & $5.63305 \mathrm{e}-14$ & $8.6475 \mathrm{e}-17$ & $8.6475 \mathrm{e}-17$ \\
$\left\|F\left(x_{k}\right)\right\|_{2}$ & $1.3365 \mathrm{e}-4$ & $9.35871 \mathrm{e}-15$ & $9.7017 \mathrm{e}-45$ & $1.5685 \mathrm{e}-134$ \\
\hline
\end{tabular}


Example 3. Solving the following two-point nonlinear boundary-value problem of ODE with exact solution:

$$
\left\{\begin{array}{l}
y^{\prime \prime}(x)=\sqrt{1+y^{\prime 2}} \\
y(0)=1, \quad y(1)=\frac{1}{2}\left(e+e^{-1}\right)
\end{array} .\right.
$$

The exact solution for this problem of ODE (37) is $y(x)=\frac{1}{2}\left(e^{-x}+e^{x}\right)$. We now find the numerical solutions using the present method and compare them with the exact solution $y(x)$.

By the finite difference method, partitioning the interval $[0,1]$ :

$x_{0}=0<x_{1}<x_{2}<\cdots<x_{n-1}<x_{n}, \quad y_{i+1}=y_{i}+h, h=1 / n$.

Let $y_{0}=y(0)=1, y_{1}=\left(x_{1}\right), \cdots, y_{n-1}=y\left(x_{n-1}\right)$, and $y_{n}=y\left(x_{n}\right)=0.5\left(e^{-1}+e\right)$. And using the numerical differential formula for the second derivative $y_{i}^{\prime \prime}=\frac{y_{i-1}-2 y_{i}+y_{i+1}}{h^{2}}$, and the first derivative $y_{i}^{\prime}=\frac{y_{i+1}-y_{i}}{h},(i=1,2, \cdots, n-1)$, we take $n=10$ herein, and hence obtain the following system of nonlinear equations with nine variables:

$$
y_{i-1}-2 y_{i}+y_{i+1}-h \sqrt{h^{2}+\left(y_{i+1}-y_{i}\right)^{2}}=0, \quad i=1,2, \cdots, 9,
$$

where $y^{(0)}=(0,0,0,0,0,0,0,0,0)^{\mathrm{T}}$ is an initial value. We obtain the approximate numerical solutions of this problem with the method (33) (iteration number $k=5$ ):

$$
\begin{aligned}
y^{*}= & (1.00412287612219 \cdots, 1.01834640006185 \cdots, 1.04286614560037 \cdots, \\
& 1.07798462092560 \cdots, 1.12411585747856 \cdots, 1.18179111195371 \cdots, \\
& 1.25166573656183 \cdots, 1.33452729205395 \cdots, 1.43130499800870 \cdots)^{\prime} .
\end{aligned}
$$

Comparison of the numerical results of the boundary-value problem of ODE (37) and the exact solutions $y\left(x_{i}\right)$ are shown in Table 3 , in which $x_{i}$ refers to the nodes.

The error comparisons of the numerical solutions and exact solutions at different nodes $x_{i}$ for solving the problem of ODE (37) are shown in Figure 1.

Table 3. Numerical comparison results for the problem of ODE (37).

\begin{tabular}{cccc}
\hline$x_{i}$ & Numerical solutions $y^{*}$ & Exact solutions $y\left(x_{i}\right)$ & The errors $\left\|y\left(x_{i}\right)-y^{*}\right\|$ \\
\hline 0.1 & $1.00412287612219 \ldots$ & $1.0050041680558035039 \ldots$ & $8.812 \mathrm{e}-04$ \\
0.2 & $1.01834640006185 \ldots$ & $1.0200667556190758933 \ldots$ & $1.720 \mathrm{e}-03$ \\
0.3 & $1.04286614560037 \ldots$ & $1.0453385141288604742 \ldots$ & $2.472 \mathrm{e}-03$ \\
0.4 & $1.07798462092560 \ldots$ & $1.0810723718384549485 \ldots$ & $3.087 \mathrm{e}-03$ \\
0.5 & $1.12411585747856 \ldots$ & $1.1276259652063806981 \ldots$ & $3.510 \mathrm{e}-03$ \\
0.6 & $1.18179111195371 \ldots$ & $1.1854652182422675821 \ldots$ & $3.674 \mathrm{e}-03$ \\
0.7 & $1.25166573656183 \ldots$ & $1.2551690056309430243 \ldots$ & $3.503 \mathrm{e}-03$ \\
0.8 & $1.33452729205395 \ldots$ & $1.3374349463048447184 \ldots$ & $2.907 \mathrm{e}-03$ \\
0.9 & $1.43130499800870 \ldots$ & $1.4330863854487745356 \ldots$ & $1.781 \mathrm{e}-03$ \\
\hline
\end{tabular}




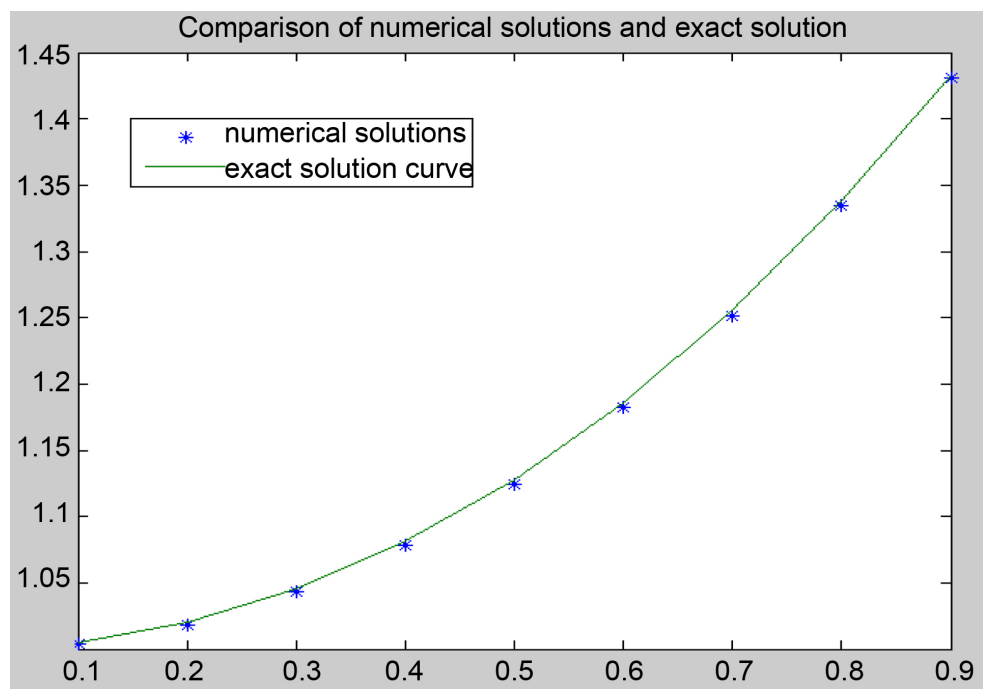

Figure 1. Error comparisons for solving the problem of ODE (37).

\section{Conclusion}

In this paper, we construct a group of iterative methods with cubic convergence for the systems of nonlinear equations by using the Gauss-Legendre quadrature formula. $\mathrm{Nu}$ merical results we gave are in consistence with the theoretical analysis, and meanwhile they also demonstrate that the presented scheme is efficient and feasible to solve systems of nonlinear equations and to solve two-point boundary-value problems of nonlinear ordinary differential equations.

\section{Acknowledgements}

The work is supported by the Science and Technology Program of Beijing Municipal Commission of Education (No. KM201511417012).

\section{References}

[1] Ortega, J.M. and Rheinboldt, W.G. (1970) Iterative Solution of Nonlinear Equations in Several Variables. Academic Press, New York.

[2] Traub, J.F. (1964) Iterative Methods for the Solution of Equations. Prentice-Hall, Englewood Cliffs.

[3] Noor, M.A. and Noor, K.I. (2006) Improved Iterative Methods for Solving Nonlinear Equations. Applied Mathematics and Computation, 183, 774-779. https:/doi.org/10.1016/j.amc.2006.05.084

[4] Chun, C. (2006) A New Ityerative Method for Solving Nonlinear Equations. Applied Mathematics and Computation, 178, 415-422. https:/doi.org/10.1016/j.amc.2005.11.055

[5] Weerakoon, S. and Fernando, T.G.I. (2000) A Variant of Newton's Method with Accelerated Third-Order Convergence. Applied Mathematics Letters, 13, 87-93. https:/doi.org/10.1016/S0893-9659(00)00100-2

[6] Darvishi, M.T. and Barati, A. (2007) A Third-Order Newton-type Method to Solve Systems of Nonlinear Equations. Applied Mathematics and Computation, 187, 630-635. https:/doi.org/10.1016/j.amc.2006.08.080 
[7] Frontini, M. and Sormani, E. (2004) Third-Order Methods from Quadrature Formulae for Solving Systems of Nonlinear Equations. Applied Mathematics and Computation, 149, 771782. https:/doi.org/10.1016/S0096-3003(03)00178-4

[8] Cordero, A. and Torregrosa, J.R. (2007) Variants of Newtons Method Using Fifth-Order Quadrature Formulas. Applied Mathematics and Computation, 190, 686-698. https:/doi.org/10.1016/j.amc.2007.01.062

[9] Noor, M.A. and Wasteem, M. (2009) Some Iterative Methods for Solving a System of Nonlinear Equations. Computers and Mathematics with Applications, 57, 101-106. https:/doi.org/10.1016/j.camwa.2008.10.067

[10] Hafiz, M.A. and Bahgat, M.S.M. (2012) An Efficient Two-Step Iterative Method for Solving System of Nonlinear Equations. Journal of Mathematics Research, 4, 28-34.

[11] Khirallah, M.Q. and Hafiz, M.A. (2012) Novel Three Order Methods for Solving a System of Nonlinear Equations. Bulletin of Mathematical Sciences \& Applications, 1, 1-14. https:/doi.org/10.18052/www.scipress.com/BMSA.2.1

[12] Guan, Z. and Lu, J.F. (1998) Numerical Analysis. High Education Press, Beijing.

Submit or recommend next manuscript to SCIRP and we will provide best service for you:

Accepting pre-submission inquiries through Email, Facebook, LinkedIn, Twitter, etc. A wide selection of journals (inclusive of 9 subjects, more than 200 journals)

Providing 24-hour high-quality service User-friendly online submission system

Fair and swift peer-review system Efficient typesetting and proofreading procedure

Display of the result of downloads and visits, as well as the number of cited articles Maximum dissemination of your research work

Submit your manuscript at: http://papersubmission.scirp.org/

Or contact jamp@scirp.org 